\title{
IR finite graviton propagators in de Sitter spacetime
}

\author{
Mir Faizal ${ }^{1, \mathrm{a}}$, Sudhaker Upadhyay ${ }^{2, \mathrm{~b}}$, Bhabani Prasad Mandal ${ }^{2, \mathrm{c}}$ \\ ${ }^{1}$ Department of Physics and Astronomy, University of Lethbridge, Lethbridge, AB T1K 3M4, Canada \\ ${ }^{2}$ Department of Physics, Banaras Hindu University, Varanasi 221005, India
}

Received: 14 June 2015 / Accepted: 8 March 2016 / Published online: 7 April 2016

(C) The Author(s) 2016. This article is published with open access at Springerlink.com

\begin{abstract}
The graviton propagator diverges in certain gauges in de Sitter spacetime. We address this problem in this work by generalizing the infinitesimal BRST transformations in de Sitter spacetime to finite field-dependent BRST (FFBRST) transformations. These FFBRST transformations are a symmetry of the classical action, but they do not leave the path integral measure invariant for the graviton theory in de Sitter spacetime. Due to the non-trivial Jacobian of such a finite transformation the path integral measure changes and hence the FFBRST transformation is capable of relating theories in two different gauges. We explicitly construct the FFBRST transformation which relates the theory with a diverging graviton two-point function to a theory with an infrared finite graviton. The FFBRST transformation thus establishes that the divergence in a graviton two-point function may be only a gauge artifact.
\end{abstract}

\section{Introduction}

The observations from type I supernovae indicate that our universe has a positive cosmological constant and may approach de Sitter spacetime asymptotically [1,3-6]. The de Sitter spacetime is also relevant in inflationary cosmology [710]. Inflaton fields corresponding to open strings have been studied in brane-antibrane models $[11,12]$ and $D 3 / D 7$ systems $[13,14]$, and the inflaton fields corresponding to closed strings have been studied in Kähler moduli $[15,16]$ and fiber inflation [17]. However, in all these models the realization of inflation depends crucially on the uplifting mechanism for de Sitter moduli stabilization [18]. This uplifting mechanism occurs in the presence of $D 3$-branes. It may be noted that even the Wilson line approach crucially depends on the uplifting mechanism for de Sitter moduli stabilization [19].

\footnotetext{
a e-mail: mirfaizalmir@gmail.com

b e-mail: sudhakerupadhyay@gmail.com

c e-mail: bhabani.mandal@gmail.com
}

Due to the relevance of de Sitter spacetime to inflation, it is important to study perturbative quantum gravity in de Sitter spacetime. However, the graviton propagator in de Sitter spacetime found by Antoniadis et al. suffered from IR divergences $[60,65]$. In fact, these IR divergences occur in the covariant gauge for certain choices of the gauge parameters, $\beta=-n(n+3) / 3$ with $n=1,2,3 \ldots$ [22]. However, it is also possible to construct an IR finite graviton propagator $[25,63,64]$. So, there are strong indications to assume that the IR divergence that occurs in the propagator by Antoniadis et al. is a gauge artifact. This is supported by the fact that the free graviton propagator in covariant gauge is equivalent to the IR finite graviton propagator [26]. However, in that analysis the role of interactions was not considered. What really needs to be demonstrated is that the generating functionals for different values of the parameter $\beta$ are related to one another. We argue that the IR divergent graviton propagators with $\beta=-n(n+3) / 3$ are related to the IR finite graviton propagators with other values of $\beta$. However, to show that explicitly, we will need a formalism to connect the generating functionals for the graviton propagators in the covariant gauge with different values of the parameter $\beta$. As the Euclidean approach has been used for a calculation of different propagators in de Sitter spacetime [27], including the graviton propagator [28], we will also use the Euclidean approach for calculating the graviton propagator. So, we will obtain the function on a four-dimensional sphere, and these Green functions are related to the Feynman propagator in the de Sitter spacetime through analytic continuation. It may be noted that we could also have used the planar patch of Lorentzian de Sitter spacetime for performing these calculations, however, the advantage of using the Euclidean approach is that it is easier to perform the FFBRST transformations in this approach. We will use the Euclidean vacuum as the vacuum state for performing these calculations [29].

The FFBRST transformation [30] was constructed systematically by integrating the usual BRST transformation [31]. Such generalized BRST transformations have the same 
form and properties as the usual BRST transformations, except these do not leave the path integral measure invariant. The non-trivial Jacobian enables such a formulation to connect theories with different effective actions, hence FFBRST transformations have found an enormous number of applications in various branches of high energy physics [30,32-47]. A similar generalization with the same motivation and goal has also been done recently in a slightly different manner [4850]. In this work we extend the FFBRST formulation [30] in de Sitter spacetime and construct an appropriate finite fielddependent parameter to relate the generating functionals corresponding to the effective theories with graviton propagator for various values of $\beta$. It may be noted that even though we build this formalism motivated by the IR divergences in de Sitter spacetime, this formalism is very general and can be used to relate a generating functional for the graviton propagator with any arbitrary value of $\beta$. It may also be noted that there are real IR divergences that occur in the ghost propagator in de Sitter spacetime. However, modes responsible for these divergences do not contribute to loop diagrams in computations of the scattering amplitudes in perturbative quantum gravity and can thus be neglected [51]. It is possible to construct an effective IR finite ghost propagator for de Sitter spacetime utilizing the FFBRST transformation. In this connection we would like to comment that the gaugeon formulation [52-57], which also connects different effective actions in perturbative quantum gravity $[58,59]$, could be another possibility to construct theories with an IR finite graviton propagator. However, the gaugeon formalism has certain drawbacks, in that one needs to introduce unphysical gaugeon fields in the theory and later extra conditions are required to extract the physical states.

It may be noted that there are various issues that are related the IR divergences in the graviton propagator. Furthermore, there are also several problems with the average gauges in de Sitter space and any space with linearizion instabilities [60]. It has also been argued that the main problem with certain values of the gauge parameter is that for these values of the gauge parameter logarithmic divergences rather than power law divergences occur $[61,62]$. The power law divergences get automatically subtracted for the allowed values of the gauge parameter. In fact, it has been demonstrate using this line of argument that certain IR divergences also occur for the allowed values of the gauge parameter $[63,64]$. Furthermore, IR divergences which appear in certain gauges have the local form of a gauge transformation, but they need not be a symmetry of the theory because the needed gauge transformation diverges at infinity and therefore invalidates the usual integration by parts and discarding of surface terms is needed to prove invariance even of the classical action [6568]. Even though we have neglected such terms in our paper by dropping a total divergence, however, we would like to point out that there are many non-trivial issues relating to the occurrence of such divergences. It may be noted that even though there are various different sources of IR divergences, in this paper, we will not address many of these issues. We will rather demonstrate that a graviton propagator in a certain gauge, in which a certain kind of IR divergences occurs, can be related to the graviton propagator in a different gauge where such IR divergences do not occur. This can be done using the FFBRST transformations, as the FFBRST transformations are a symmetry of the generating functional and not of the effective action, which is obtained by adding the gauge fixing and ghosts terms to the original action. In fact, it is this property of the FFBRST transformation that has made it possible to use the FFBRST transformation for analyzing various interesting physical systems [30,32-47]. Thus, motivated by such uses of the FFBRST transformations, we will analyze the occurrence of a certain kind of IR divergences in this paper.

In this paper, we first study the perturbative quantum gravity on curved space time where we particularly emphasize the de Sitter spacetime. The effective action of perturbative quantum gravity on de Sitter spacetime respects a fermionic rigid BRST invariance. The BRST symmetry further generalizes by making the parameter finite and field-dependent following the techniques of Ref. [30]. The FFBRST transformation generalized in such a way leads to a non-trivial Jacobian for a functional measure. We show that for a particular choice of the finite field-dependent parameter the Jacobian relates the gauge parameters, stimulating IR divergent and IR finite graviton propagators. So, in Sect. 2, we analyze the perturbative quantum gravity in de Sitter spacetime, and in Sect. 3 we study the FFBRST transformation in de Sitter spacetime. Then in Sect. 4 we relate the IR divergent graviton twopoint function to the IR finite graviton propagators using the FFBRST transformations. In the final section we summarize the results.

\section{Perturbative quantum gravity}

Let us first of all start by analyzing the perturbative quantum gravity in de Sitter spacetime. The line element for de Sitter spacetime, which is a contracting and expanding threesphere, is given by

$$
\begin{aligned}
\mathrm{d} s^{2}= & -\mathrm{d} t^{2}+\frac{1}{H^{2}} \cosh ^{2}(H t) \\
& \times\left[\mathrm{d} \chi^{2}+\sin ^{2} \chi\left(\mathrm{d} \theta^{2}+\sin \theta^{2} \mathrm{~d} \phi^{2}\right)\right],
\end{aligned}
$$

where $H$ is the Hubble constant. In terms of the variable $\tau \equiv \pi / 2-i H t$, the line element gets the following form:

$\mathrm{d} s^{2}=H^{-2}\left\{\mathrm{~d} \tau^{2}+\sin ^{2} \tau\left[\mathrm{d} \chi^{2}+\sin ^{2} \chi\left(\mathrm{d} \theta^{2}+\sin \theta^{2} \mathrm{~d} \phi^{2}\right)\right]\right\}$, 
which is the line element of a four-dimensional sphere of radius $H^{-1}$. Now we can set $H^{2}=1$. The Lagrangian density of pure gravity in de Sitter spacetime is given by

$\mathcal{L}_{d s}=\sqrt{-g^{(f)}}\left(R^{(f)}-6\right)$,

where we have set $16 \pi G=1$. Here $g_{\mu \nu}^{(f)}$ is the full metric and $R^{(f)}$ is the curvature corresponding to it. This Lagrangian is invariant under general coordinate transformations,

$\delta_{\Lambda} g_{\mu \nu}^{(f)}=£_{\Lambda} g_{\mu \nu}^{(f)}$

where $£_{\Lambda} g_{\mu \nu}^{(f)}=\Lambda^{\tau} \nabla_{\tau} g_{\mu \nu}^{(f)}+g_{\mu \tau}^{(f)} \nabla_{\nu} \Lambda^{\tau}+g_{\mu \tau}^{(f)} \nabla_{\mu} \Lambda^{\tau}$, is the Lie derivative. Now we can expand $g_{\mu \nu}^{(f)}$ in terms of a fixed background metric, $g_{\mu \nu}$, and a small perturbation around it, $h_{\mu \nu}$. We have

$g_{\mu \nu}^{(f)}=g_{\mu \nu}+h_{\mu \nu}$.

Now we can also expand the Lagrangian for gravity with a cosmological constant in terms of this fixed background metric and a small perturbation around it. Furthermore, this small perturbation is regarded as the quantum field to be quantized in perturbative quantum gravity. It may be noted that this Lagrangian will contain infinitely many terms, because the original Lagrangian contained the inverse of the metric in it. Now as $g_{\mu \nu}$ is fixed, the transformation of $g_{\mu \nu}^{(f)}$ will be attributed to $h_{\mu \nu}, \delta_{\Lambda} h_{\mu \nu}=£_{\Lambda} g_{\mu \nu}^{(f)}=£_{\Lambda} g_{\mu \nu}+£_{\Lambda} h_{\mu \nu}$. Now to the first order in $\Lambda_{\mu}$, the Lagrangian for perturbative quantum gravity will be invariant to all orders in $h_{\mu \nu}$, under the following transformation:

$\delta_{\Lambda} h_{\mu \nu}=\nabla_{\mu} \Lambda_{v}+\nabla_{\nu} \Lambda_{\mu}+£_{\Lambda} h_{\mu \nu}$,

where the Lie derivative $£_{\Lambda} h_{\mu \nu}$ is given by $£_{\Lambda} h_{\mu \nu}=$ $\Lambda^{\tau} \nabla_{\tau} h_{\mu \nu}+h_{\mu \tau} \nabla_{\nu} \Lambda^{\tau}+h_{\nu \tau} \nabla_{\mu} \Lambda^{\tau}$. The resulting Lagrangian density for the linearized gravity is written, after dropping a total divergence,

$$
\begin{aligned}
\mathcal{L}_{d s}= & \mathcal{L}_{f}+\mathcal{L}_{i n t} \\
= & \sqrt{-g}\left[\frac{1}{2} \nabla_{\mu} h^{\mu \eta} \nabla^{\nu} h_{\nu \eta}-\frac{1}{4} \nabla_{\mu} h_{\nu \eta} \nabla^{\mu} h^{\nu \eta}\right. \\
& +\frac{1}{4}\left(\nabla^{\mu} h-2 \nabla^{v} h^{\mu}{ }_{\nu}\right) \nabla_{\mu} h \\
& \left.-\frac{1}{2}\left(h_{\mu \nu} h^{\mu \nu}+\frac{1}{2} h^{2}\right)\right]+\mathcal{L}_{i n t},
\end{aligned}
$$

with $h=h_{\mu}^{\mu}$, and $\mathcal{L}_{\text {int }}$ is the interaction part of the Lagrangian.

To quantize the theory we need to break this gauge invariance for canonical quantization. Here this is achieved by choosing a general (covariant) gauge fixing condition for this Lagrangian, thus $\left[\nabla^{v} h_{\mu \nu}-k \nabla_{\mu} h\right]=0$,

where $k \neq 1$. It may be noted that for $k=1$, the gauge redundancies are not fully removed, and therefore usually $k$ is written as $1+\beta^{-1}$ for a finite value of $\beta$. The gauge fixing condition can be incorporated at a quantum level by the addition of a gauge fixing term to the original Lagrangian,

$\mathcal{L}_{g f}=\sqrt{-g} b^{\mu}\left[\nabla^{v} h_{\mu \nu}-\left(1+\beta^{-1}\right) \nabla_{\mu} h\right]+\sqrt{-g} \frac{\alpha}{2} b^{\mu} b_{\mu}$

We can obtain the ghost term corresponding to this gauge fixing term by first taking the gauge transformation of the gauge fixing condition, then replacing all the gauge parameters with ghosts, and finally contracting the quantity thus obtained with anti-ghosts. Thus, the ghost term corresponding to this gauge fixing term can be written as

$$
\begin{aligned}
\mathcal{L}_{g h}= & \sqrt{-g} \bar{c}^{\mu} \nabla^{\nu}\left[\nabla_{\mu} c_{\nu}+\nabla_{\nu} c_{\mu}-2\left(1+\beta^{-1}\right) g_{\mu \nu} \nabla^{\tau} c_{\tau}\right. \\
& +\left(£_{c} h_{\mu \nu}-\left(1+\beta^{-1}\right) g_{\mu \nu} g^{\left.\left.\tau \rho_{£_{c}} h_{\tau \rho}\right)\right],}\right.
\end{aligned}
$$

where $£_{c} h_{\mu \nu}$ is given by $£_{c} h_{\mu \nu}=c^{c} \nabla_{c} h_{\mu \nu}+h_{\mu \sigma} \nabla_{\nu} c^{\sigma}+$ $h_{\nu \sigma} \nabla_{\mu} c^{\sigma}$. Now the sum of the deformed Lagrangian for gravity, the gauge fixing term, and the ghost term is invariant under the following BRST transformations:

$s b_{\mu}=0, \quad s h_{\mu \nu}=\nabla_{\mu} c_{\nu}+\nabla_{\nu} c_{\mu}+£_{c} h_{\mu \nu}$,

$s \bar{c}^{\mu}=b^{\mu}, \quad s c_{\mu}=c^{\nu} \nabla_{\nu} c_{\mu}$.

It may be noted that the invariance of the sum of the non-local Lagrangian for gravity, along with the gauge fixing term and the ghost term under the BRST transformation, follows from the nilpotency of the BRST transformations, $s \mathcal{L}=0$. This is because the sum of the gauge fixing term and the ghost term can be written as a total BRST variation,

$\mathcal{L}_{g f}+\mathcal{L}_{g h}=s\left[\sqrt{-g} \bar{c}^{\mu}\left[\nabla^{\mu} h_{\mu \nu}-\left(1+\beta^{-1}\right) \nabla_{\mu} h+\frac{\alpha}{2} b_{\mu}\right]\right]$.

It is well known that the graviton two-point function is IR divergent for $\beta=-n(n+3) / 3$ [22]. So, we take the initial gauge fixing condition as

$G_{1}[h]=\left[\nabla^{v} h_{\mu \nu}-\left(1+\beta_{1}\right)^{-1} \nabla_{\mu} h\right]=0$,

where

$\beta_{1}=-\frac{n(n+3)}{3}+\epsilon$,

and we take the limit $\epsilon \rightarrow 0$, at the end of our calculation. In the next section, we demonstrate that it is possible to gauge away this IR divergence using the FFBRST transformation. Hence, an IR finite propagator is obtained even after taking 
the limit. These IR divergences are only gauge artifacts. We take our final gauge fixing condition to be

$G_{2}[h]=\left[\nabla^{v} h_{\mu \nu}-\left(1+\beta_{2}\right)^{-1} \nabla_{\mu} h\right]=0$,

where

$\beta_{2} \neq-\frac{n(n+3)}{3}$.

Now we can write the sum of the gauge fixing and ghost terms for the initial gauge fixing condition as

$$
\begin{aligned}
& \mathcal{L}_{1 g f}+\mathcal{L}_{1 g h} \\
& \quad=s\left[\sqrt{-g} \bar{c}^{\mu}\left[\nabla^{\mu} h_{\mu \nu}-\left(1+\beta_{1}^{-1}\right) \nabla_{\mu} h+\frac{\alpha}{2} b_{\mu}\right]\right],
\end{aligned}
$$

and we can also write the sum of the gauge fixing and ghost terms for the final gauge fixing condition as

$$
\begin{aligned}
& \mathcal{L}_{2 g f}+\mathcal{L}_{2 g h} \\
& \quad=s\left[\sqrt{-g} \bar{c}^{\mu}\left[\nabla^{\mu} h_{\mu \nu}-\left(1+\beta_{2}^{-1}\right) \nabla_{\mu} h+\frac{\alpha}{2} b_{\mu}\right]\right] .
\end{aligned}
$$

Now we can write the total Lagrangian as the sum of the original Lagrangian, the gauge fixing, and ghost terms.

$$
\begin{aligned}
& \mathcal{L}_{1}=\mathcal{L}_{d s}+\mathcal{L}_{1 g f}+\mathcal{L}_{1 g h}, \\
& \mathcal{L}_{2}=\mathcal{L}_{d s}+\mathcal{L}_{2 g f}+\mathcal{L}_{2 g h} .
\end{aligned}
$$

We can neglect the interaction part of the Lagrangian and write the equation of motions, from these Lagrangians as follows:

$$
\begin{aligned}
& L_{\mu \nu\left(\beta_{1}\right)}^{\sigma \lambda} h_{\sigma \lambda}=0, \\
& L_{\mu \nu\left(\beta_{2}\right)}^{\sigma \lambda} h_{\sigma \lambda}=0,
\end{aligned}
$$

where

$$
\begin{aligned}
& L_{\mu \nu\left(\beta_{1}\right)}^{\sigma \lambda} h_{\sigma \lambda} \\
& =\frac{1}{2} \nabla^{\tau} \nabla_{\tau} h_{\mu \nu}-\left(\frac{1}{2}-\frac{1}{2 \alpha}\right)\left(\nabla_{\mu} \nabla_{\tau} h_{\nu}^{\tau}+\nabla_{\nu} \nabla_{\tau} h_{\mu}^{\tau}\right) \\
& \quad+\left(\frac{1}{2}-\frac{\beta_{1}+1}{\alpha \beta_{1}}\right) \nabla_{\mu} \nabla_{\nu} h+\left(\frac{\left(\beta_{1}+1\right)^{2}}{\alpha \beta_{1}^{2}}-\frac{1}{2}\right) g_{\mu \nu} \nabla^{\tau} \nabla_{\tau} h \\
& \quad+\left(\frac{1}{2}-\frac{1+\beta_{1}}{\alpha \beta_{1}}\right) g_{\mu \nu} \nabla^{\tau} \nabla^{\rho} h_{\tau \rho}-h_{\mu \nu}-\frac{1}{2} g_{\mu \nu} h, \\
& L_{\mu \nu\left(\beta_{2}\right)}^{\sigma \lambda} h_{\sigma \lambda} \\
& =\frac{1}{2} \nabla^{\tau} \nabla_{\tau} h_{\mu \nu}-\left(\frac{1}{2}-\frac{1}{2 \alpha}\right)\left(\nabla_{\mu} \nabla_{\tau} h_{\nu}^{\tau}+\nabla_{\nu} \nabla_{\tau} h_{\mu}^{\tau}\right) \\
& \quad+\left(\frac{1}{2}-\frac{\beta_{2}+1}{\alpha \beta_{2}}\right) \nabla_{\mu} \nabla_{\nu} h+\left(\frac{\left(\beta_{2}+1\right)^{2}}{\alpha \beta_{2}^{2}}-\frac{1}{2}\right) g_{\mu \nu} \nabla^{\tau} \nabla_{\tau} h \\
& \quad+\left(\frac{1}{2}-\frac{1+\beta_{2}}{\alpha \beta_{2}}\right) g_{\mu \nu} \nabla^{\tau} \nabla^{\rho} h_{\tau \rho}-h_{\mu \nu}-\frac{1}{2} g_{\mu \nu} h .
\end{aligned}
$$

So, the graviton two-point function for the two different gauges can be defined as

$L_{\mu \nu\left(\beta_{1}\right)}^{\tau \rho} G_{\tau \rho \tau^{\prime} \rho^{\prime}}^{\left(\beta_{1}\right)}\left(x, x^{\prime}\right)=\delta_{\mu \nu \tau^{\prime} \rho^{\prime}}\left(x, x^{\prime}\right)$,
$L_{\mu \nu\left(\beta_{2}\right)}^{\tau \rho} G_{\tau \rho \tau^{\prime} \rho^{\prime}}^{\left(\beta_{2}\right)}\left(x, x^{\prime}\right)=\delta_{\mu \nu \tau^{\prime} \rho^{\prime}}\left(x, x^{\prime}\right)$.

This graviton two-point function in the final gauge is IR finite $[25,63,64]$, whereas the graviton two-point function in the initial gauge diverges for $\epsilon \rightarrow 0$ [22]. It may be noted that the exact expression for the graviton propagator will depend on the exact choice of the gauge, however, the important point is that such a graviton propagator is IR finite in a certain gauge. We now will proceed to show that it is possible to construct a particular FFBRST transformation which takes the generating functional in the initial gauge to that in the final gauge. Thus the FFBRST takes the theory with an IR divergent graviton propagator to the theory with an IR finite graviton propagator. This indicates that the IR divergence of graviton propagator is a gauge artifact. It may be noted that even though we have performed our analysis to relate the generating functionals in the de Sitter spacetime, this analysis is very general and can be used to relate generating functionals with different values of $\beta$ in any curved spacetime.

\section{FFBRST transformation}

To construct the FFBRST transformation [30] for the theory of quantum gravity in de Sitter spacetime we first write the usual BRST transformation characterized by an infinitesimal Grassmann parameter $\delta \Lambda$ as follows:

$\delta_{b} \Phi^{i}(x)=s \Phi^{i}(x) \delta \Lambda$,

where $\Phi^{i}(x)=\left(h^{\mu \nu}(x), c^{\mu}(x), \bar{c}^{\mu}(x), b^{\mu}(x)\right)$ is the set of all fields in the theory. It may be noted that the invariance under BRST transformation is not affected by $\delta \Lambda$ being (i) finite or infinitesimal, (ii) field dependent or field independent, as long as it is anticommuting and spacetime independent. This motivates the generalization of the BRST transformations to finite field-dependent BRST transformations. This is done by first making the infinitesimal parameter $\delta \Lambda$ field dependent, and introducing an arbitrary parameter $\kappa$, such that $0 \leq \kappa \leq 1$. Then all the fields are made to depend on $\kappa$, in such a way that $\Phi^{i}(x, \kappa=0)$ are the initial fields, and $\Phi^{i}(x, \kappa=1)$ are the transformed fields. Now we can write the field-dependent infinitesimal BRST transformations as

$$
\begin{aligned}
& s h_{\mu \nu}=\left(\nabla_{\mu} c_{\nu}+\nabla_{\nu} c_{\mu}+£_{(c)} h_{\mu \nu}\right) \Theta^{\prime}[\Phi(x, \kappa)], \\
& s c^{\mu}=c_{\nu} \nabla^{v} c^{\mu} \Theta^{\prime}[\Phi(x, \kappa)], \\
& s \bar{c}^{\mu}=b^{\mu} \Theta^{\prime}[\Phi(x, \kappa)], \\
& s b^{\mu}=0,
\end{aligned}
$$


where $\Theta^{\prime}[\Phi(x, \kappa)]$ is an infinitesimal but field-dependent parameter. Now the finite field-dependent BRST (FFBRST) transformations can be constructed by integrating such infinitesimal field-dependent BRST transformations [58],

$$
\begin{aligned}
& f h_{\mu \nu}=\left(\nabla_{\mu} c_{\nu}+\nabla_{\nu} c_{\mu}+\mathfrak{f}_{(c)} h_{\mu \nu}\right) \Theta[\Phi(x)], \\
& f c^{\mu}=c_{\nu} \nabla^{v} c^{\mu} \Theta[\Phi(x)], \\
& f \bar{c}^{\mu}=b^{\mu} \Theta[\Phi(x)], \\
& f b^{\mu}=0,
\end{aligned}
$$

where the finite field-dependent parameter is given by

$\Theta[\Phi(x)]=\int_{0}^{1} \mathrm{~d} \kappa \Theta^{\prime}[\Phi(x, \kappa)]$.

So, the FFBRST transformation can be written as

$$
f \Phi^{i}(x)=\Phi^{i}(x, \kappa=1)-\Phi^{i}(x, \kappa=0)=s\left[\Phi^{i}(x)\right] \Theta[\Phi] .
$$

This FFBRST transformation is a symmetry of the effective action only but not of the functional measure, because the path integral measure changes under the FFBRST transformation to a local functional of the fields. Suppose the Jacobian of the path integral measure under such a transformation is written

$$
\begin{aligned}
\mathcal{D} \Phi & =J(\kappa) \mathcal{D} \Phi(\kappa), \\
& =J(\kappa+\mathrm{d} \kappa) \mathcal{D} \Phi(\kappa+\mathrm{d} \kappa) .
\end{aligned}
$$

The transformation from $\Phi(\kappa)$ to $\Phi(\kappa+\mathrm{d} \kappa)$ is an infinitesimal one and one has for its Jacobian

$\frac{J(\kappa)}{J(\kappa+\mathrm{d} \kappa)}=\int \mathrm{d}^{4} x \sum_{\Phi} \pm \frac{\delta \Phi^{i}(x, \kappa+\mathrm{d} \kappa)}{\delta \Phi^{i}(x, \kappa)}$,

where $\sum_{\Phi}$ sums over all the fields in the measure and the \pm sign refers to the cases of fields $\Phi^{i}(x, \kappa)$ being bosonic or fermionic in nature. Now utilizing Taylor's expansion the infinitesimal change in the Jacobian is calculated by [58]

$\frac{1}{J} \frac{\mathrm{d} J}{\mathrm{~d} \kappa}=-\int \mathrm{d}^{4} y \sum_{\Phi}\left[ \pm s \Phi^{i}(y, \kappa) \frac{\delta \Theta^{\prime}[\Phi]}{\delta \Phi^{i}(y, \kappa)}\right]$.

Now the Jacobian, $J(\kappa)$, can be replaced (within the functional integral) by

$J(\kappa) \rightarrow \exp \left[i S_{1}[\Phi(x, \kappa), \kappa]\right]$

where $S_{1}[\Phi(x), \kappa]$ is a local functional of the fields, if the following condition is satisfied:

$\frac{1}{J} \frac{\mathrm{d} J}{\mathrm{~d} \kappa}-i \frac{\mathrm{d} S_{1}[\Phi(x, \kappa), \kappa]}{\mathrm{d} \kappa}=0$.
Therefore, by constructing an appropriate $\Theta^{\prime}$, we are able calculate the non-trivial (local) Jacobian which extends the effective action by a term $S_{1}$.

\section{Recovering IR finite the graviton propagators}

In the previous section, we have analyzed the FFBRST transformation for the de Sitter spacetime in a general gauge. In this section, we explicitly use the results of the previous section to demonstrate that the IR divergence in the graviton two-point function can be gauged away. The FFBRST transformation for perturbative quantum gravity corresponding to the BRST transformation are written as given by Eq. (25). Now to show the connection between IR divergence of the graviton propagators and IR finiteness of the graviton propagators we derive a specific $\Theta[\Phi]$ constructed from the following infinitesimal field-dependent parameter:

$\Theta^{\prime}[\Phi]=\int \mathrm{d}^{4} x \sqrt{-g} \bar{c}^{\mu}\left[\left(\beta_{1}^{-1}-\beta_{2}^{-1}\right) \nabla_{\mu} h\right]$.

Using Eq. (30) along with Eq. (33), we calculate the infinitesimal change in Jacobian as follows:

$$
\begin{aligned}
\frac{1}{J(\kappa)} \frac{\mathrm{d} J(\kappa)}{\mathrm{d} \kappa}= & \int \mathrm{d}^{4} x \sqrt{-g}\left[-b^{\mu}\left(-\beta_{1}^{-1}+\beta_{2}^{-1}\right) \nabla_{\mu} h\right. \\
& -\bar{c}^{\mu} \nabla^{\nu}\left[2\left(-\beta_{1}^{-1}+\beta_{2}^{-1}\right) g_{\mu \nu} \nabla^{\tau} c_{\tau}\right. \\
& \left.\left.+\left(-\beta_{1}^{-1}+\beta_{2}^{-1}\right) g_{\mu \nu} g^{\tau \rho} \mathfrak{£}_{c} h_{\tau \rho}\right]\right] .
\end{aligned}
$$

Now we make an ansatz for the local functional $S_{1}[\Phi, \kappa]$, which can be written as

$$
\begin{aligned}
S_{1}[\Phi, \kappa]= & \int \mathrm{d}^{4} x \sqrt{-g}\left[\xi_{1}(\kappa) b^{\mu}\left(\beta_{1}^{-1}\right) \nabla_{\mu} h\right. \\
& +\xi_{2}(\kappa) b^{\mu}\left(\beta_{2}^{-1}\right) \nabla_{\mu} h+\xi_{3}(\kappa) \bar{c}^{\mu} \nabla^{\nu}\left(\beta_{1}^{-1}\right) \\
& \times g_{\mu \nu} \nabla^{\tau} c_{\tau}+\xi_{4}(\kappa) \bar{c}^{\mu} \nabla^{\nu}\left(\beta_{2}^{-1}\right) g_{\mu \nu} \nabla^{\tau} c_{\tau} \\
& +\xi_{5}(\kappa) \bar{c}^{\mu} \nabla^{\nu}\left(\beta_{1}^{-1}\right) g_{\mu \nu} g^{\tau \rho_{£_{c}}} h_{\tau \rho} \\
& \left.+\xi_{6}(\kappa) \bar{c}^{\mu} \nabla^{\nu}\left(\beta_{2}^{-1}\right) g_{\mu \nu} g^{\tau \rho} \mathfrak{£}_{c} h_{\tau \rho}\right],
\end{aligned}
$$

where $\xi_{i}(i=1,2, \ldots, 6)$ are arbitrary constant $\kappa$-dependent parameters which can be evaluated from the essential condition given by Eq. (32). The essential condition given by Eq. (32), along with Eqs. (34) and (35), leads to the following differential equations:

$\xi_{1}^{\prime}-1=0, \quad \xi_{2}^{\prime}+1=0, \quad \xi_{3}^{\prime}-2=0$,

$\xi_{4}^{\prime}+2=0, \quad \xi_{5}^{\prime}-1=0, \quad \xi_{6}^{\prime}+1=0$.

The particular solutions of above equations satisfying initial boundary conditions $\xi_{i}(\kappa=0)=0$ are

$\xi_{1}=1, \quad \xi_{2}=-1, \quad \xi_{3}=2$,

$\xi_{4}=-2, \quad \xi_{5}=1, \quad \xi_{6}=-1$. 
With these values of the parameters the local functional comprising Jacobian is given by

$$
\begin{aligned}
& S_{1}[\Phi, \kappa] \\
& \quad=\int \mathrm{d}^{4} x \sqrt{-g}\left[\kappa b^{\mu}\left(\beta_{1}^{-1}\right) \nabla_{\mu} h-\kappa b^{\mu}\left(\beta_{2}^{-1}\right) \nabla_{\mu} h\right. \\
& \quad+2 \kappa \bar{c}^{\mu} \nabla^{\nu}\left(\beta_{1}^{-1}\right) g_{\mu \nu} \nabla^{\tau} c_{\tau}-2 \kappa \bar{c}^{\mu} \nabla^{\nu}\left(\beta_{2}^{-1}\right) g_{\mu \nu} \nabla^{\tau} c_{\tau} \\
& \quad+\kappa \bar{c}^{\mu} \nabla^{\nu}\left(\beta_{1}^{-1}\right) g_{\mu \nu} g^{\tau \rho} \mathfrak{£}_{c} h_{\tau \rho} \\
& \left.\quad-\kappa \bar{c}^{\mu} \nabla^{\nu}\left(\beta_{2}^{-1}\right) g_{\mu \nu} g^{\tau \rho_{£_{c}}} h_{\tau \rho}\right],
\end{aligned}
$$

which vanishes at $\kappa=0$. However, at $\kappa=1$ it translates into

$$
\begin{aligned}
& S_{1}[\Phi, 1] \\
& =\int \mathrm{d}^{4} x \sqrt{-g}\left[b^{\mu}\left(\beta_{1}^{-1}\right) \nabla_{\mu} h-b^{\mu}\left(\beta_{2}^{-1}\right) \nabla_{\mu} h\right. \\
& \quad+2 \bar{c}^{\mu} \nabla^{\nu}\left(\beta_{1}^{-1}\right) g_{\mu \nu} \nabla^{\tau} c_{\tau}-2 \bar{c}^{\mu} \nabla^{\nu}\left(\beta_{2}^{-1}\right) g_{\mu \nu} \nabla^{\tau} c_{\tau} \\
& \left.\quad+\bar{c}^{\mu} \nabla^{\nu}\left(\beta_{1}^{-1}\right) g_{\mu \nu} g^{\tau \rho_{c}} h_{\tau \rho} \bar{c}^{\mu} \nabla^{\nu}\left(\beta_{2}^{-1}\right) g_{\mu \nu} g^{\tau \rho} £_{c} h_{\tau \rho}\right],
\end{aligned}
$$

which implies that the Jacobian can be written as $\exp i S_{1}[\Phi, 1]$. Now, this Jacobian changes the effective action of the path integral as follows:

$S_{1 g f}+S_{1 g h}+S_{1}[\Phi, 1]=S_{2 g f}+S_{2 g h}$,

where

$$
\begin{aligned}
& S_{1 g f}+S_{1 g h}=\int \mathrm{d}^{4} x \sqrt{-g}\left(b^{\mu}\left[\nabla^{\nu} h_{\mu \nu}-\left(1+\beta_{1}^{-1}\right) \nabla_{\mu} h\right]\right. \\
& +\frac{\alpha}{2} b^{\mu} b_{\mu}+\bar{c}^{\mu} \nabla^{\nu}\left[\nabla_{\mu} c_{\nu}+\nabla_{\nu} c_{\mu}-2\left(1+\beta_{1}^{-1}\right) g_{\mu \nu} \nabla^{\tau} c_{\tau}\right. \\
& \left.\left.+\left(\mathfrak{£}_{c} h_{\mu \nu}-\left(1+\beta_{1}^{-1}\right) g_{\mu \nu} g^{\tau \rho} \mathfrak{£}_{c} h_{\tau \rho}\right)\right]\right), \\
& S_{2 g f}+S_{2 g h}=\int \mathrm{d}^{4} x \sqrt{-g}\left(b^{\mu}\left[\nabla^{\nu} h_{\mu \nu}-\left(1+\beta_{2}^{-1}\right) \nabla_{\mu} h\right]\right. \\
& +\frac{\alpha}{2} b^{\mu} b_{\mu}+\bar{c}^{\mu} \nabla^{\nu}\left[\nabla_{\mu} c_{\nu}+\nabla_{\nu} c_{\mu}-2\left(1+\beta_{2}^{-1}\right) g_{\mu \nu} \nabla^{\tau} c_{\tau}\right. \\
& \left.\left.+\left(\mathfrak{£}_{c} h_{\mu \nu}-\left(1+\beta_{2}^{-1}\right) g_{\mu \nu} g^{\tau \rho} \mathfrak{f}_{c} h_{\tau \rho}\right)\right]\right) \text {. }
\end{aligned}
$$

We can write the action sum of the original classical action, the gauge fixing term, and the ghost term for the initial gauge as

$$
S_{1 T}=\int \mathrm{d}^{4} x\left[\mathcal{L}_{d s}+\mathcal{L}_{1 g f}+\mathcal{L}_{1 g h}\right],
$$

and we can write the action sum of the original classical action, the gauge fixing term, and the ghost term for the final gauge as

$S_{2 T}=\int \mathrm{d}^{4} x\left[\mathcal{L}_{d s}+\mathcal{L}_{2 g f}+\mathcal{L}_{2 g h}\right]$.

Now we can take the limit $\epsilon \rightarrow 0$ for this transformed action and obtain $\lim _{\epsilon \rightarrow 0} S_{1 T}+\lim _{\epsilon \rightarrow 0} S_{1}[\Phi, 1]=\lim _{\epsilon \rightarrow 0} S_{2 T}$.

The two-point function obtained from the action $S_{1 T}$ is IR divergent in the limit $\epsilon \rightarrow 0$, and the two-point function obtained from the action $S_{2 T}$ is IR finite in the limit $\epsilon \rightarrow 0$. Hence, in the limit $\epsilon \rightarrow 0$, the action $S_{1}[\Phi, 1]$ also produces IR divergent contributions and these contributions exactly cancel the IR divergence coming from $S_{1 T}$ as follows:

$$
\begin{aligned}
& \lim _{\epsilon \rightarrow 0} \int \mathcal{D} \Phi \mathcal{O}[\Phi]_{2} e^{i S_{1 T}[\Phi]} \\
& \stackrel{\text { FFBRST }}{\longrightarrow} \lim _{\epsilon \rightarrow 0} \int \mathcal{D} \Phi \mathcal{O}[\Phi]_{2} e^{i S_{2 T}[\Phi]},
\end{aligned}
$$

where $\mathcal{O}[\Phi]_{2}$ is a two-point composite operator. We can neglect the interactions, and calculate the relation between Green's functions. Now if $G_{\mu \nu \tau^{\prime} \rho^{\prime}}\left(x, x^{\prime}\right)$ is the contribution to the Green function coming from $S_{1}[\Phi, 1]$, then we can write

$$
\begin{aligned}
& \lim _{\epsilon \rightarrow 0}\left[G_{\mu \nu \tau^{\prime} \rho^{\prime}}\left(x, x^{\prime}\right)+G_{\mu \nu \tau^{\prime} \rho^{\prime}}^{\left(\beta_{1}\right)}\left(x, x^{\prime}\right)\right] \\
& \quad=\lim _{\epsilon \rightarrow 0}\left[G_{\mu \nu \tau^{\prime} \rho^{\prime}}^{\left(\beta_{2}\right)}\left(x, x^{\prime}\right)\right] .
\end{aligned}
$$

Hence, it has been possible to gauge away these IR divergences in the graviton two-point function in de Sitter spacetime using an FFBRST transformation. This implies that these IR divergences are only gauge artifacts. It may be noted as the gauge fixing and the ghost terms are not affected by including interactions in the action. In fact, it has been demonstrated that the FFBRST transformation are a symmetry of the generating functional, and so it would be possible to relate the two generating functionals even after the interactions have been added. It may be noted that, as the FFBRST transformations relate the full generating functional, they have been applied to various interesting physical systems [30,32-47]. Thus, even if we do not neglect the interactions, we have

$$
\begin{aligned}
& S_{I T}=S f+S_{\mathrm{int}}+S_{1 g f}+S_{1 g h} ; \\
& S_{2 T}=S f+S_{\mathrm{int}}+S_{2 g f}+S_{2 g h} ;
\end{aligned}
$$

where the $S_{\text {int }}$ are the interactions in the perturbative quantum gravity. So, we can take the limit $\epsilon \rightarrow 0$ for the actions, even after taking the interactions into account,

$\lim _{\epsilon \rightarrow 0} S_{1 T}+\lim _{\epsilon \rightarrow 0} S_{1}[\Phi, 1]=\lim _{\epsilon \rightarrow 0} S_{2 T}$.

Now for any operator $\mathcal{O}[\Phi]$, we can formally write

$$
\begin{aligned}
& \lim _{\epsilon \rightarrow 0} \int \mathcal{D} \Phi \mathcal{O}[\Phi] e^{i S_{1 T}[\Phi]} \\
& \stackrel{F F B R S T}{\longrightarrow} \lim _{\epsilon \rightarrow 0} \int \mathcal{D} \Phi \mathcal{O}[\Phi] e^{i S_{2 T}[\Phi]} .
\end{aligned}
$$


Hence, at least formally we can argue that such an IR divergence will not occur even in loop calculations. However, as we were interested in demonstrating the relation between two Green's functions in this paper, we have explicitly only demonstrated this for the Green functions.

\section{Conclusion}

In this paper, we have analyzed perturbative quantum gravity on de Sitter spacetime. The BRST and FFBRST transformations for the perturbative quantum gravity were explicitly constructed in de Sitter spacetime. The FFBRST transformations were used to relate the generating functionals with different values of the parameter $\beta$. We construct an appropriate finite field-dependent parameter such that the Jacobian contribution of the path integral measure relates the graviton propagator with an IR divergence to the IR finite graviton propagator. Thus, it was argued that it might be possible that a certain kind of IR divergence in the graviton propagator is only a gauge artifact. However, we would like to point out that there are arguments to try to argue that the removal of such divergences is only an artifact of the regularization procedure $[61,62]$. Since the spacetime noncommutativity changes the IR behavior of quantum field theories $[69,70]$, and perturbative quantum gravity has been studied on noncommutative spacetime [71-75], it would also be interesting to analyze the IR divergences in de Sitter spacetime in noncommutative spacetime.

Open Access This article is distributed under the terms of the Creative Commons Attribution 4.0 International License (http://creativecomm ons.org/licenses/by/4.0/), which permits unrestricted use, distribution, and reproduction in any medium, provided you give appropriate credit to the original author(s) and the source, provide a link to the Creative Commons license, and indicate if changes were made. Funded by SCOAP ${ }^{3}$.

\section{References}

1. A.G. Riess et al., Astron. J. 116, 1009 (1998)

2. S. Perlmutter et al., Nature 391, 51 (1998)

3. A.G. Riess et al., Astron. J. 118, 2668 (1999)

4. S. Perlmutter et al., Astrophys. J. 517, 565 (1999)

5. A.G. Riess et al., Astrophys. J. 560, 49 (2001)

6. J.L. Tonry et al., Astrophys. J. 594, 1 (2003)

7. K. Bamba, G. Cognola, S.D. Odintsov, S. Zerbini, Phys. Rev. D 90, 023525 (2014)

8. A. del Rio, J. Navarro-Salas, Phys. Rev. D 89, 084037 (2014)

9. D. Seery, JCAP. 0905, 021 (2009)

10. S. Dubovsky, L. Senatore, G. Villadoro, JHEP. 0904, 118 (2009)

11. C.P. Burgess, M. Majumdar, D. Nolte, F. Quevedo, G. Rajesh, R.J. Zhang, JHEP. 0107, 047 (2001)

12. S. Kachru, R. Kallosh, A. Linde, J. Maldacena, L. McAllister, S.P. Trivedi, JCAP. 0310, 013 (2003)
13. K. Dasgupta, C. Herdeiro, S. Hirano, R. Kallosh, Phys. Rev. D 65, $126002(2002)$

14. C.P. Burgess, J.M. Cline, M. Postma, JHEP. 0903, 058 (2009)

15. J.P. Conlon, F. Quevedo, JHEP. 0601, 146 (2006)

16. J.R. Bond, L. Kofman, S. Prokushkin, P.M. Vaudrevange, Phys. Rev. D 75, 123511 (2007)

17. M. Cicoli, C.P. Burgess, F. Quevedo, JCAP. 0903, 013 (2009)

18. S. Krippendorf, F. Quevedo, JHEP. 0911, 039 (2009)

19. A. Avgoustidis, D. Cremades, F. Quevedo, Gen. Rel. Grav. 39, 1203 (2007)

20. I. Antoniadis, J. Iliopoulos, T.N. Tomaras, Phys. Rev. Lett. 56, 1319 (1986)

21. I. Antoniadis, E. Mottola, J. Math. Phys. 32, 1037 (1991)

22. B. Allen, Phys. Rev. D 34, 3670 (1986)

23. A. Higuchi, R.H. Weeks, Class. Quant. Grav. 20, 3005 (2003)

24. A. Higuchi, D. Marolf, I.A. Morrison, Class. Quant. Grav. 28, $245012(2011)$

25. R.P. Bernar, L.C.B. Crispino, A. Higuchi, Phys. Rev. D 90, 024045 (2014)

26. M. Faizal, A. Higuchi, Phys. Rev. D 85, 12402 (2012)

27. B. Allen, T. Jacobson, Commun. Math. Phys. 103, 669 (1986)

28. A. Higuchi, S.S. Kouris, Class. Quantum Grav. 18, 4317 (2001)

29. G. Gibbons, S.W. Hawking, Phys. Rev. D 15, 2738 (1977)

30. S.D. Joglekar, B.P. Mandal, Phys. Rev. D 51, 1919 (1995)

31. M. Chaichian, N.F. Nelipa, Introduction to Gauge Field Theories (Springer, Berlin Heidelberg, 2012), p. 332

32. S. Upadhyay, S.K. Rai, B.P. Mandal, J. Math. Phys. 52, 022301 (2011)

33. S.D. Joglekar, A. Misra, Int. J. Mod. Phys. A 15, 1453 (2000)

34. S. Upadhyay, B.P. Mandal, Eur. Phys. J. C 72, 2065 (2012)

35. S. Upadhyay, B.P. Mandal, Ann. Phys. 327, 2885 (2012)

36. S. Upadhyay, B.P. Mandal, Mod. Phys. Lett. A 25, 3347 (2010)

37. S. Upadhyay, B. P. Mandal, Prog. Theor. Exp. Phys. 053B04 (2014)

38. S. Upadhyay, M.K. Dwivedi, B.P. Mandal, Int. J. Mod. Phys. A 28, 1350033 (2013)

39. R. Banerjee, B.P. Mandal, Phys. Lett. B 488, 27 (2000)

40. R. Banerjee, B. Paul, S. Upadhyay, Phys. Rev. D 88, 065019 (2013)

41. R. Banerjee, S. Upadhyay, Phys. Lett. B 734, 369 (2014)

42. S. Upadhyay, D. Das, Phys. Lett. B 733, 63 (2014)

43. S. Upadhyay, EPL 104, 61001 (2013)

44. S. Upadhyay, Phys. Lett. B 727, 293 (2013)

45. S. Upadhyay, Ann. Phys. 340, 110 (2014)

46. M. Faizal, S. Upadhyay, B.P. Mandal, Phys. Lett. B 738, 201 (2014)

47. M. Faizal, B.P. Mandal, S. Upadhyay, Phys. Lett. B 721, 159 (2013)

48. P.M. Lavrov, O. Lechtenfeld, Phys. Lett. B 725, 382 (2013)

49. P.Y. Moshin, A.A. Reshetnyak, Nucl. Phys. B 888, 92 (2014)

50. P.Y. Moshin, A.A. Reshetnyak, Int. J. Mod. Phys. A 30, 1550021 (2015)

51. M. Faizal, A. Higuchi, Phys. Rev. D 78, 067502 (2008)

52. K. Yokoyama, Prog. Theor. Phys. 51, 1956 (1974)

53. S. Upadhyay, EPL 105, 21001 (2014)

54. K. Yokoyama, Prog. Theor. Phys. 59, 1699 (1978)

55. S. Upadhyay, B.P. Mandal, Prog. Theor. Exp. Phys. 053 B04 (2014)

56. K. Yokoyama, Prog. Theor. Phys. 60, 1167 (1978)

57. K. Yokoyama, Phys. Lett. B 79, 79 (1978)

58. S. Upadhyay, Ann. Phys. 344, 290 (2014)

59. S. Upadhyay, Eur. Phys. J. C 74, 2737 (2014)

60. S.P. Miao, N.C. Tsamis, R.P. Woodard, J. Math. Phys. 50, 122502 (2009)

61. S.P. Miao, N.C. Tsamis, R.P. Woodard, J. Math. Phys. 51, 072503 (2010)

62. S.P. Miao, N.C. Tsamis, R.P. Woodard, J. Math. Phys. 52, 122301 (2011)

63. P.J. Mora, N.C. Tsamis, R.P. Woodard, J. Math. Phys. 52, 122301 (2011) 
64. P.J. Mora, N.C. Tsamis, R.P. Woodard, J. Math. Phys. 53, 2122502 (2012)

65. H. Bondi, M.G.J. van der Burg, A.W.K. Metzner, Proc. R. Soc. Lond. A 269, 21 (1962)

66. R.K. Sachs, Proc. R. Soc. Lond. A 270, 103 (1962)

67. A. Strominger, JHEP 1407, 152 (2014)

68. R.P. Woodard, arXiv: 1506.04252

69. R. Horvat, A. Ilakovac, J. Trampetic, J. You, JHEP 1112, 081 (2011)
70. H. Grosse, H. Steinacker, M. Wohlgenannt, JHEP 0804, 023 (2008)

71. M. Faizal, J. Phys. A 44, 402001 (2011)

72. M. Faizal, Phys. Lett. B 705, 120 (2011)

73. M. Faizal, Mod. Phys. Lett. A 27, 1250075 (2012)

74. J.W. Moffat, Phys. Lett. B 491, 345 (2000)

75. J.W. Moffat, Phys. Lett. B 493, 142 (2000) 